\title{
Collisionless magnetic reconnection in the presence of a sheared velocity field
}

\author{
M. Faganello, ${ }^{1}$ F. Pegoraro, ${ }^{2}$ F. Califano, ${ }^{2}$ and L. Marradi ${ }^{2,3}$ \\ ${ }^{1}$ École Polytechnique, LPP, Palaiseau, 91128 France \\ ${ }^{2}$ Department of Physics, University of Pisa and CNISM, Pisa, 56127 Italy \\ ${ }^{3}$ Université de Nice Sophia Antipolis, CNRS, Observatoire de la Côte d'Azur, 06304 Nice, France
}

(Received 18 March 2010; accepted 26 April 2010; published online 4 June 2010)

\begin{abstract}
The linear theory of magnetic field lines reconnection in a two-dimensional configuration in the presence of a (Kelvin-Helmholtz stable) sheared velocity field is investigated within a single fluid model, where the onset of magnetic field line reconnection is made possible by the effect of electron inertia in the so called large $\Delta^{\prime}$ regime. (C) 2010 American Institute of Physics.
\end{abstract}

[doi:10.1063/1.3430640]

\section{INTRODUCTION}

Inhomogeneous current distributions and sheared velocity fields are ubiquitous features of laboratory and space plasmas. In fact, together with pressure gradients in the presence of curved magnetic field lines or gravity forces, they represent two of the most important sources of instability in a magnetized plasma. Velocity field shear may lead to the onset of the Kelvin-Helmholtz instability ${ }^{1,2}$ that causes the formation of velocity vortices that, in the nonlinear phase of the instability, roll up and pair. ${ }^{3-5}$ Current layers on the contrary tend to tear apart, causing magnetic field lines to reconnect. ${ }^{6-8}$ The Kelvin-Helmholtz instability is mainly a hydrodynamic, ideal instability while magnetic reconnection is related to the evolution of the magnetic field in the plasma. Nevertheless, the fact that at low frequencies and large spatial scales in a high conductivity plasma, the dynamics of the magnetic field and of the plasma are tightly linked by the so called "frozen-in" condition brings the evolution of the velocity and of the magnetic field together.

In principle, magnetic reconnection is a local instability that should be expected to come into play once faster, large scale magnetic instabilities occurring on the Alfvèn time scale have developed in the plasma. However, even if the breaking of the field lines occurs only locally, the resulting restructuring of the magnetic connection between the plasma fluid elements is global and, in addition, if externally driven, magnetic reconnection can occur on time scales that are not very different from the ideal Alfvèn times and from the hydrodynamical time scales. ${ }^{9-13}$

For these reasons in the literature, the interplay between the Kelvin-Helmholtz instability and magnetic field line reconnection has long since been studied with two main aims: investigate the stabilizing effect of a velocity shear field on the development of magnetic reconnection in configurations that are Kelvin-Helmholtz stable ${ }^{14-18}$ on the one hand and, on the other, investigate how the evolution of the KelvinHelmholtz instability can stretch the magnetic field lines and lead to field line reconnection, provided the resulting field tension be weak enough not to inhibit the onset and the evolution of the Kelvin-Helmholtz instability. ${ }^{1,2}$ The KelvinHelmholtz instability can drive magnetic reconnection in two different ways. If the magnetic field has an inversion line [we refer, for simplicity, to a two-dimensional (2-D) configuration, see below], the fluid velocity field, modified by the linear evolution of Kelvin-Helmholtz, forces the magnetic lines to reconnect and to follow the evolution imposed by the Kelvin-Helmholtz instability. ${ }^{18-22}$ On the contrary, if the magnetic field initially has no inversion line, magnetic reconnection cannot occur during the linear phase of the KelvinHelmholtz instability. However, the nonlinear evolution of the Kelvin-Helmholtz vortices is able to roll up and stretch the magnetic lines, producing inversion regions and leading to the formation of reconnection unstable current layers. $^{12,13,21,23-26}$

The modification of the reconnection process that occurs in the presence of a Kelvin-Helmholtz stable, sheared velocity field has been mainly investigated in 2-D configurations, ${ }^{14-18}$ where the sheared velocity field and the inhomogeneous magnetic field have coincident null lines in the $x-y$ plane, while $z$ is an ignorable coordinate. In these configurations, the magnetic field component along $z$, if present, is taken to be homogeneous over the spatial scales of interest. Furthermore, fluid regimes were generally considered where the local violation of the perfect conductivity condition was caused by the effect of a small but finite resistivity. ${ }^{14-18}$ This is not an accurate model when we aim at studying almost dissipationless plasma regimes where a kinetic plasma description should be introduced, at least in the regions were the current layers form and where, even if a fluid description is still adopted, resistivity is not the main effect that violates the perfect conductivity condition.

In the present paper, we revisit analytically the linear theory of magnetic field lines reconnection in a 2-D configuration (i.e., we consider perturbations that do not depend on the $z$ coordinate) within a single fluid model where, however, the onset of magnetic field line reconnection is made possible by the effect of electron inertia ${ }^{27,28}$ instead of resistivity. This magnetohydrodynamic (MHD) model with electron inertia has a number of interesting differences with respect to the resistive model. First it maintains the Hamiltonian, i.e., dissipation free, character of high temperature dilute plasma regimes, ${ }^{29}$ as would be the case for a full kinetic description. 
Second, it strongly discriminates between the "reconnecting" (large $\Delta^{\prime}$ ) regime from the "tearing" (small $\Delta^{\prime}$, also called constant- $\psi$ ) regime. In fact, in the MHD plus electron inertia description, the growth rate of the tearing instability turns out to be too small to be of relevance as it scales, ${ }^{30,31}$ in the absence of a velocity field, proportionally to $\left(d_{e} / L\right)^{3}$. Here, $L$ is a macroscopic scale length and $d_{e} \ll L$ is the collisionless inertial skin depth. On the contrary, in the large $\Delta^{\prime}$ regime, the instability growth rate scales ${ }^{27,31}$ as $d_{e} / L$. Thus in this article we will consider the large $\Delta^{\prime}$ regime only.

We will show that in a magnetic inverted equilibrium that is Kelvin-Helmholtz stable, the growth rate of the collisionless magnetic reconnection instability is modified by the presence of a sheared velocity field. In particular, although the large $\Delta^{\prime}$ growth rate still scales as $d_{e} / L$ (reconnecting regime), reconnection is partially stabilized by the sheared velocity field. Furthermore, we will show that even in the large $\Delta^{\prime}$ regime, the reconnection instability is fully stabilized by the velocity field when the slope of the equilibrium velocity profile equals the slope of the Alfvén velocity profile at the magnetic null line. As shown analytically in Refs. 14, 15, and 17 and numerically in Refs. 16 and 18, when the fluid velocity profile is steeper than the Alfvén velocity profile a transition to the ideal Kelvin-Helmholtz instability occurs. In this case, reconnection is not an instability but is simply driven by the far faster ideal instability. ${ }^{18-22}$

\section{PLASMA MODEL}

\section{A. Governing equations and equilibrium configuration}

We start from the dissipationless MHD set of equations in the incompressible limit with electron inertia included in Ohm's law. We write this set in the form

$$
\begin{aligned}
& {\left[\frac{\partial \mathbf{U}}{\partial t}+(\mathbf{U} \cdot \nabla) \mathbf{U}\right]=-\nabla P+(\nabla \times \mathbf{B}) \times \mathbf{B},} \\
& E+\frac{\mathbf{U} \times \mathbf{B}}{c}=d_{e}^{2}\left[\frac{\partial \mathbf{J}}{\partial t}+(\mathbf{U} \cdot \nabla) \mathbf{J}\right], \\
& \nabla \times \mathbf{E}=-\frac{\partial \mathbf{B}}{\partial t}, \quad \nabla \times \mathbf{B}=\mathbf{J}, \\
& \nabla \cdot \mathbf{U}=0, \quad \nabla \cdot \mathbf{B}=0,
\end{aligned}
$$

where dimensionless units are used normalized on the magnetic equilibrium spatial scale length $L_{B}$, on the Alfvén velocity $U_{A}=B_{0} / \sqrt{4 \pi \rho_{0}}$ with $\rho_{0}$ the constant mass density, and on the corresponding Alfvén time $\tau_{A}=L_{B} / U_{A}$. Here, $d_{e}$ is the normalized electron collisionless skin depth.

We consider a one-dimensional equilibrium configuration where the three-dimensional magnetic and velocity fields depend on the inhomogeneity coordinate $x$ only

$$
\mathbf{B}_{\text {eq }}=B_{y}(x) \hat{y}+B_{z}(x) \hat{z}, \quad \mathbf{U}_{\text {eq }}=U_{y}(x) \hat{y}+U_{z}(x) \hat{z} .
$$

Since we are interested here in the investigation of how an equilibrium velocity field can modify the development in the reconnection instability, we consider a velocity jump $\Delta U_{y / z}$ that is lower than the Alfvén velocity $U_{A, y / z}$. In this case, the magnetic tension is strong enough to prevent the development of the Kelvin-Helmholtz instability. ${ }^{1,2}$

\section{B. Linearized equations}

Now we consider small perturbations of the equilibrium fields which we Fourier transform along the homogeneous directions $y$ and $z$. Thus, the linearized equations, obtained by neglecting the second order terms, are

$$
\begin{aligned}
& (\gamma+i \alpha G)\left(W^{\prime \prime}-\alpha^{2} W\right)-i \alpha G^{\prime \prime} W=i \alpha F\left(\psi^{\prime \prime}-\alpha^{2} \psi\right) \\
& \quad-i \alpha F^{\prime \prime} \psi, \\
& (\gamma+i \alpha G) \psi-i \alpha F W=d_{e}^{2}\left\{(\gamma+i \alpha G)\left(\psi^{\prime \prime}-\alpha^{2} \psi\right)-i \alpha F^{\prime \prime} W\right\},
\end{aligned}
$$

where $\gamma$ is the growth rate, $\alpha=k L_{B}$ is the normalized module of the wave vector, $k^{2}=k_{y}^{2}+k_{z}^{2}$, and

$$
F(x)=\frac{\mathbf{k} \cdot \mathbf{B}_{\mathrm{eq}}(\mathbf{x})}{k B_{0}}, \quad G(x)=\frac{\mathbf{k} \cdot \mathbf{U}(\mathbf{x})_{\mathrm{eq}}(\mathbf{x})}{k U_{A}} .
$$

Note that the incompressibility limit allows us to describe the linear evolution using only the perturbed magnetic flux $\psi=B_{\text {pert }, x} / B_{0}$ and the $x$ component $W=U_{\text {pert }, x} / U_{A}$ of the perturbed velocity, and to take into account equilibrium variation only along the wave vector direction. In fact, $F(x)$ and $G(x)$ measure the magnetic and velocity equilibrium variations along the wave vector direction, respectively. The incompressibility condition (4) is well satisfied if there is a strong guide magnetic field perpendicular to the plane where the instability develops (i.e., the plane defined by the $x$-direction and the wave vector $\mathbf{k}$ ).

\section{BOUNDARY LAYER APPROACH}

For the sake of simplicity, we assume that the magnetic field has only one inversion point at $x=0$, i.e., $F(0)=0$. Furthermore, we assume that the velocity profile is monotonic and that $G(0)=0$. This last condition can always be satisfied by a change in the reference frame.

Thus, if we neglect all the terms proportional to the small parameter $d_{e}^{2}$ in Eqs. (6) and (7), for $\gamma \ll 1$, the corresponding ideal MHD equations are singular at the inversion point $x=0$. As usual, we consider a boundary layer approach and divide the whole space in an external region, where the ideal MHD equations hold, and in an internal layer across the singular point $x=0$, where the small $d_{e}^{2}$ terms are able to remove the singularity.

\section{A. External region}

In the external region, we can neglect all terms proportional to the small parameters $d_{e}^{2}$ and $\gamma$. Thus Eqs. (6) and (7) correspond to stationary ideal MHD equations

$$
i \alpha G\left(W^{\prime \prime}-\alpha^{2} W\right)-i \alpha G^{\prime \prime} W=i \alpha F\left(\psi^{\prime \prime}-\alpha^{2} \psi\right)-i \alpha F^{\prime \prime} \psi,
$$




$$
i \alpha G \psi-i \alpha F W=0 .
$$

These equations correspond to the usual zeroth order expansion of the of the Hydromagnetic equations analyzed in Refs. 14 and 17. The internal solution must match the external solution at all orders in the small expansion parameters $\gamma$ and $d_{e}^{2}$, but it is sufficient to consider the zeroth order of the external solution in order to obtain a physical solution.

Following the standard approach, ${ }^{7}$ we estimate the value of the internal solutions $\psi, W$ considering the limit of Eq. (10) for $x \rightarrow 0$. This "use of the frozen-in law for internal solutions" leads to

$$
\psi \sim \frac{i \alpha F^{\prime}(0) \epsilon}{\gamma+i \alpha G^{\prime}(0) \epsilon} W,
$$

where $\epsilon \ll 1$ is the width of the internal region. This scaling is valid in both the external and internal region. ${ }^{14,15,17}$

\section{B. Internal region}

Let us consider an internal layer of width $\epsilon \ll 1$ across the singular point $x=0$. In this "very thin" region, the derivatives of $\psi$ and $W$ became so large that the $d_{e}^{2}$ terms in Eqs. (6) and (7) can no longer be neglected.

We consider a "stretched" variable $\xi=x / \epsilon$ and the Taylor series in $x=0$ of the equilibrium fields $G(x)=G^{\prime}(0) x$ $+1 / 2 G^{\prime \prime}(0) x^{2}+O\left(x^{3}\right)$ and $F(x)=F^{\prime}(0) x+1 / 2 F^{\prime \prime}(0) x^{2}+O\left(x^{3}\right)$. Equations (6) and (7) now read

$$
\begin{gathered}
{\left[\frac{\gamma}{\alpha F^{\prime}(0) \epsilon}+i \frac{G^{\prime}(0)}{F^{\prime}(0)} \xi+\frac{1}{2} i \frac{G^{\prime \prime}(0)}{F^{\prime}(0)} \epsilon \xi^{2}\right] \frac{\partial^{2} W}{\partial \xi^{2}}-i \frac{G^{\prime \prime}(0)}{F^{\prime}(0)} \epsilon W} \\
=\left[i \xi+\frac{1}{2} i \frac{F^{\prime \prime}(0)}{F^{\prime}(0)} \epsilon \xi^{2}\right] \frac{\partial^{2} \psi}{\partial \xi^{2}}-i \frac{F^{\prime \prime}(0)}{F^{\prime}(0)} \epsilon \psi+O\left(\epsilon^{2}\right), \\
{\left[\frac{\gamma}{\alpha F^{\prime}(0) \epsilon}+i \frac{G^{\prime}(0)}{F^{\prime}(0)} \xi+\frac{1}{2} i \frac{G^{\prime \prime}(0)}{F^{\prime}(0)} \epsilon \xi^{2}\right] \psi-i \xi W} \\
-\frac{1}{2} i \frac{F^{\prime \prime}(0)}{F^{\prime}(0)} \epsilon \xi^{2} W \\
=\frac{d_{e}^{2}}{\epsilon^{2}}\left\{\left[\frac{\gamma}{\alpha F^{\prime}(0) \epsilon}+i \frac{G^{\prime}(0)}{F^{\prime}(0)} \xi+\frac{1}{2} i \frac{G^{\prime \prime}(0)}{F^{\prime}(0)} \epsilon \xi^{2}\right] \frac{\partial^{2} \psi}{\partial \xi^{2}}\right. \\
\left.-i \frac{F^{\prime \prime}(0)}{F^{\prime}(0)} \epsilon W\right\}+O\left(\epsilon^{2}\right) .
\end{gathered}
$$

As usual, we suppose that inside the internal layer the mode amplitude variation along $x$ is far more important than those along $y$ and $z$, i.e., $\partial / \partial \xi \gg \alpha$.

\section{Matching condition}

The solutions obtained in the external and internal region must overlap in the limit $|x| \rightarrow 0$ and $|\xi| \rightarrow \infty$. We define the standard matching parameter ${ }^{7} \Delta^{\prime}$ as

$$
\Delta^{\prime}=\left.\frac{1}{\psi_{\mathrm{ext}}(x)} \frac{\partial \psi_{\mathrm{ext}}(x)}{\partial x}\right|_{0_{-}} ^{0^{+}}=\left.\frac{1}{\epsilon \psi_{\mathrm{int}}(\xi)} \frac{\partial \psi_{\mathrm{int}}(\xi)}{\partial \xi}\right|_{-\infty} ^{+\infty} .
$$

The value of the parameter $\Delta^{\prime}$ is set by the external ideal solution and thus it can be strongly modified by the occur- rence of an equilibrium velocity field. ${ }^{14,17}$ However, if the large scale magnetic and velocity field are such that $G(x)$ $\propto F(x)$, the value of $\Delta^{\prime}$ is unaffected with respect to its value $\Delta_{U=0}^{\prime}$ in the case of a stationary plasma. The fact that $\Delta^{\prime}$ is strongly affected by the velocity field should not appear surprising, since the magnetic field in the external region is frozen-in the plasma motion.

\section{KINK MODE}

In the case of a stationary plasma equilibrium $\mathbf{U}_{\text {eq }}=0$, the collisionless kink mode ${ }^{27,31}$ has a growth rate proportional to $d_{e}$, while the collisionless tearing mode, or so called constant- $\psi$, scales as $d_{e}^{3} e^{30,31}$ For this reason, the kink mode has attracted more attention than the tearing mode. These two regimes are usually characterized by the value of the matching parameter $\Delta^{\prime} \gg 1$ for the kink mode and $\Delta^{\prime} \sim 1$ for the tearing mode and are known as the fast growth regime $\gamma \sim\left|\alpha F^{\prime}(0) \epsilon\right|$ and the slow growth regime $\gamma \ll\left|\alpha F^{\prime}(0) \epsilon\right|$, respectively. ${ }^{17}$

In general, the collisionless tearing mode is too slow to be competitive with other plasma processes that influence the plasma dynamics. Therefore, we focus our attention on the kink mode evolution, or fast growth regime, where

$$
\left|\frac{\gamma}{\alpha F^{\prime}(0) \epsilon}\right| \sim 1
$$

Modifications resulting from the presence of a large scale flow $\mathbf{U}_{\text {eq }} \neq 0$ in Eqs. (6) and (7) are controlled by the parameter $G^{\prime}(0) / F^{\prime}(0)$ which represents the ratio of the velocity and magnetic shear at the magnetic null point.

As shown analytically in Refs. 14, 15, and 17 and numerically in Refs. 16 and 18, there can be a transition to the ideal Kelvin-Helmholtz instability (much faster than the reconnection instability) when this parameter is larger than 1. In the following we will consider the regime $\left|G^{\prime}(0) / F^{\prime}(0)\right| \lesssim 1$.

\section{A. Scaling laws}

In the internal layer and using the frozen-in condition given by Eq. (11), in the fast growth regime Eq. (15) allows us to state that

$$
\psi \sim W .
$$

At zeroth order in the small parameters $\epsilon$ and $d_{e}^{2}$, Eqs. (12) and (13) read

$$
\begin{aligned}
& {\left[\frac{\gamma}{\alpha F^{\prime}(0) \epsilon}+i \frac{G^{\prime}(0)}{F^{\prime}(0)} \xi\right] \frac{\partial^{2} W}{\partial \xi^{2}}=i \xi \frac{\partial^{2} \psi}{\partial \xi^{2}},} \\
& {\left[\frac{\gamma}{\alpha F^{\prime}(0) \epsilon}+i \frac{G^{\prime}(0)}{F^{\prime}(0)} \xi\right] \psi-i \xi W} \\
& =\frac{d_{e}^{2}}{\epsilon^{2}}\left[\frac{\gamma}{\alpha F^{\prime}(0) \epsilon}+i \frac{G^{\prime}(0)}{F^{\prime}(0)} \xi\right] \frac{\partial^{2} \psi}{\partial \xi^{2}} .
\end{aligned}
$$

The balance between the various terms of Eqs. (17) and (18) gives 


$$
\frac{\gamma}{\alpha F^{\prime}(0) \epsilon} \sim 1, \quad \xi \sim 1 \Rightarrow \frac{d_{e}^{2}}{\epsilon^{2}} \frac{\gamma}{\alpha F^{\prime}(0) \epsilon} \sim 1,
$$

and thus we have

- Internal layer width $\epsilon \sim d_{e}$ and

- Growth rate $\gamma \sim \alpha F^{\prime}(0) d_{e} \propto d_{e}$.

These scaling laws do not depend on the value of $\left|G^{\prime}(0) / F^{\prime}(0)\right|$, provided it is comparable or smaller than 1 . For this reason, these scaling laws are the same as those obtained for the collisionless kink mode in a stationary plasma. $^{27,31}$

\section{B. Small velocity shear regime}

When the velocity shear at the magnetic null point is small compared to the magnetic shear, i.e., $\left|G^{\prime}(0) / F^{\prime}(0)\right|$ $\ll 1$, it is possible to obtain the value of the growth rate in the kink regime, i.e., for $\Delta^{\prime} \gg 1$, explicitly with an expansion procedure. Equations (17) and (18) can be rewritten as

$$
\begin{aligned}
& \frac{\partial}{\partial \xi}\left(A^{2} \frac{\partial \phi}{\partial \xi}\right)=-\xi \frac{\partial^{2} \psi}{\partial \xi^{2}}, \\
& A(\psi-\xi \phi)=\frac{d_{e}^{2}}{\epsilon^{2}} \frac{\partial^{2} \psi}{\partial \xi^{2}} A,
\end{aligned}
$$

where

$$
A=\frac{\gamma}{\alpha F^{\prime}(0) \epsilon}+i \frac{G^{\prime}(0)}{F^{\prime}(0)} \xi, \quad \phi=\frac{i W}{A} .
$$

By integrating Eq. (20) we can define the new variable $X$

$$
\xi \frac{\partial \psi}{\partial \xi}-\psi=-A^{2} \frac{\partial \phi}{\partial \xi}+C_{0} \equiv X,
$$

and from Eq. (21), we have

$$
\frac{d_{e}^{2}}{\epsilon^{2}}\left(\frac{\partial^{2} X}{\partial \xi^{2}}-\frac{2}{\xi} \frac{\partial X}{\partial \xi}\right)=X+\frac{\xi^{2}}{A^{2}}\left(X-C_{0}\right) .
$$

Furthermore we can state that

$$
\left.\frac{\partial \psi}{\partial x}\right|_{0^{-}} ^{0^{+}}=\int_{0^{-}}^{0^{+}} \frac{\partial^{2} \psi}{\partial x^{2}} d x=\frac{1}{\epsilon} \int_{-\infty}^{+\infty} \frac{\partial^{2} \psi}{\partial \xi^{2}} d \xi=\frac{1}{\epsilon} \int_{-\infty}^{+\infty} \frac{1}{\xi} \frac{\partial X}{\partial \xi} d \xi .
$$

Since, as we will see, $\psi \rightarrow C_{0}$ for $|\xi| \rightarrow \pm \infty$, the usual matching condition can be rewritten as

$$
\Delta^{\prime}=\frac{1}{\epsilon C_{0}} \int_{-\infty}^{+\infty} \frac{1}{\xi} \frac{\partial X}{\partial \xi} d \xi
$$

We can thus consider the power expansions in the small parameter $\left|G^{\prime}(0) / F^{\prime}(0)\right|$

$$
X=\sum_{n=0}^{\infty} X_{n}\left[i \frac{G^{\prime}(0)}{F^{\prime}(0)}\right]^{n}=\sum_{n=0}^{\infty} X_{n} \Gamma^{n},
$$

$$
\gamma=\sum_{n=0}^{\infty} \gamma_{n}\left[i \frac{G^{\prime}(0)}{F^{\prime}(0)}\right]^{n}=\sum_{n=0}^{\infty} \gamma_{n} \Gamma^{n} .
$$

At zero order in the small parameter $\Gamma$, Eqs. (22)-(24) become

$$
\begin{aligned}
& A=\frac{\gamma_{0}}{\alpha F^{\prime}(0) \epsilon}+O(\Gamma), \\
& \frac{d_{e}^{2}}{\epsilon}\left(\frac{\partial^{2} X_{0}}{\partial \xi^{2}}-\frac{2}{\xi} \frac{\partial X_{0}}{\partial \xi}\right)=X_{0}+\frac{\alpha^{2} F^{\prime}(0)^{2} \epsilon^{2}}{\gamma_{0}^{2}} \xi^{2}\left(X_{0}-C_{0}\right) .
\end{aligned}
$$

The asymptotic behavior of the zeroth order solution is thus given by

$$
X_{0} \rightarrow C_{0}-\frac{C_{0} \gamma_{0}^{2}}{\alpha^{2} F^{\prime}(0)^{2} \epsilon^{2}} \frac{1}{\xi^{2}} \quad \text { for } \quad|\xi| \rightarrow+\infty .
$$

From the matching condition (26) we have, in the Kink regime $\Delta^{\prime} \epsilon \gg 1$,

$$
C_{0}=0, \quad X_{0}=e^{-\xi^{2} / 2}, \quad \frac{d_{e}^{2}}{\epsilon^{2}}=\frac{\alpha^{2} F^{\prime}(0)^{2} \epsilon^{2}}{\gamma_{0}^{2}}=1,
$$

and thus

- $\gamma_{0}=\alpha F^{\prime}(0) d_{e}=\gamma_{U_{\mathrm{eq}}=0}$

- $\epsilon=d_{e}$.

Note that this is the solution of the singular Sturm-Liouville problem given by Eq. (30) with $C_{0}=0$. The solution (32) has no inversion point and thus represents the fundamental state of this problem, corresponding to the smallest eigenvalue $\alpha^{2} F^{\prime}(0)^{2} \epsilon^{2} / \gamma_{0}^{2}$, and thus to the largest growth rate $\gamma_{0}$. The singularity is related to the existence of the continuous spectrum. Its contribution to the perturbation evolution is proportional to $t^{-|a|}$ and is thus negligible compared to the exponential instability. ${ }^{32-34}$

At the first order in $\Gamma$ we have

$$
\begin{aligned}
& A=\frac{\gamma_{0}}{\alpha F^{\prime}(0) \epsilon}+\left[\xi+\frac{\gamma_{1}}{\alpha F^{\prime}(0) \epsilon}\right] \Gamma+O\left(\Gamma^{2}\right) \\
& =1+\left(\xi+\frac{\gamma_{1}}{\gamma_{0}}\right) \Gamma+O\left(\Gamma^{2}\right), \\
& \frac{1}{A^{2}}=1-2\left(\xi+\frac{\gamma_{1}}{\gamma_{0}}\right) \Gamma+O\left(\Gamma^{2}\right), \\
& \frac{\partial^{2} X_{1}}{\partial \xi^{2}}-\frac{2}{\xi} \frac{\partial X_{1}}{\partial \xi}=\left(1+\xi^{2}\right) X_{1}-2 \xi^{2}\left(\frac{\gamma_{1}}{\gamma_{0}}+\xi\right) X_{0} .
\end{aligned}
$$

Once that the zeroth order solution $X_{0}$ is fixed by Eq. (32), we can determine the first order solution $X_{1}$ that satisfies the proper boundary condition given by $X_{1} \rightarrow 0$ for $|\xi| \rightarrow+\infty$

$$
X_{1}=\frac{1}{3} \xi^{3} e^{-\xi^{2} / 2}, \quad \gamma_{1}=0 .
$$

In order to obtain a correction to the stationary configuration growth rate $\gamma_{0}=\gamma_{U_{\mathrm{eq}}=0}$, we are forced to consider the second order of the expansion series in $\Gamma$. At this order, Eqs. (22)-(24) read 


$$
\begin{aligned}
& A=1+\Gamma \xi+\frac{\gamma_{2}}{\gamma_{0}} \Gamma^{2}+O\left(\Gamma^{3}\right) \\
& \frac{1}{A^{2}}=1-2 \Gamma \xi+\left(3 \xi^{2}-2 \frac{\gamma_{2}}{\gamma_{0}}\right) \Gamma^{2}+O\left(\Gamma^{3}\right), \\
& \frac{\partial^{2} X_{2}}{\partial \xi^{2}}-\frac{2}{\xi} \frac{\partial X_{2}}{\partial \xi}=\left(1+\xi^{2}\right) X_{2}-2 \xi^{3} X_{1}+\xi^{2}\left(3 \xi-2 \frac{\gamma_{2}}{\gamma_{0}}\right) X_{0} .
\end{aligned}
$$

Given the lower order solutions $X_{0}$ and $X_{1}$, we obtain the second order solution

$$
X_{2}=\frac{1}{18} \xi^{6} e^{-\xi^{2} / 2}-\frac{1}{4} \xi^{4} e^{-\xi^{2} / 2}, \quad \frac{\gamma_{2}}{\gamma_{0}}=\frac{1}{2} .
$$

Thus we obtain for the growth rate $\gamma_{U_{\mathrm{eq}}} \neq 0$

$$
\begin{aligned}
\gamma_{U_{\mathrm{eq}} \neq 0} & \sim \alpha F^{\prime}(0) d_{e}\left\{1-\left[G^{\prime}(0) / 2 F^{\prime}(0)\right]^{2}\right\} \\
& =\gamma_{U_{\mathrm{eq}}=0}\left\{1-\left[G^{\prime}(0) / 2 F^{\prime}(0)\right]^{2}\right\} .
\end{aligned}
$$

In the small velocity shear regime, the effect of the equilibrium velocity field is to provide a small reduction in the growth rate of the kink mode.

Note that for consistency, $\Gamma^{2}=\left[G^{\prime}(0) / F^{\prime}(0)\right]^{2}$ must be greater than the small parameter $\epsilon \sim d_{e}$ as we have neglected all the terms $O(\epsilon)$.

\section{Comparable shear regime}

As stated before, the scaling laws obtained in Sec. IV A do not depend on the value of $G^{\prime}(0) / F^{\prime}(0)$. Thus, the kink growth rate is proportional to $d_{e}$ also when $G^{\prime}(0) / F^{\prime}(0)$ $\lesssim 1$.

It is important to notice that there can be a transition to the ideal Kelvin-Helmholtz instability when $\left|G^{\prime}(0) / F^{\prime}(0)\right|$ $\rightarrow 1,{ }^{14-18}$ and thus a suppression of the reconnection instability.

The Kelvin-Helmholtz ideal instability is far faster than the reconnection instability. Furthermore, it has been shown that the velocity field, generated by Kelvin-Helmholtz instability, is able to force the magnetic field lines to reconnect. $^{18-22}$ This reconnection is not an instability but is induced by the ideal Kelvin-Helmholtz instability. In fact, for a resistive plasma, the rate at which it happens is independent from the value of the resistivity and is related only to the Kelvin-Helmholtz growth rate. ${ }^{18,20}$

It is also possible to show that the collisionless kink mode becomes stable for $\left|G^{\prime}(0) / F^{\prime}(0)\right| \rightarrow 1$. In fact when $\left|G^{\prime}(0) / F^{\prime}(0)\right|$ approaches 1 , the constant- $\psi$ approximation is valid also for $\Delta^{\prime} \gg 1$, as shown in the resistive case in Refs. 14 and 17. There is thus a transition from the kink mode to the slower tearing mode, which is stable for $\left|G^{\prime}(0) / F^{\prime}(0)\right|$ $\rightarrow 1$. This not only leads to a slower growth rate, but also to a total stabilization of the collisionless kink mode (see Appendix for analytical results). In order to confirm the scaling law $\gamma \propto d_{e}$ and the stabilization of the Kink mode for $\left|G^{\prime}(0) / F^{\prime}(0)\right| \rightarrow 1$, Eqs. (6) and (7) have been integrated numerically in the parameter ranges $10<\Delta^{\prime}<20,0.1<d_{e}$ $<0.5$, and $5000<S_{\eta}<10000$, where $S_{\eta}$ is the resistive
Lundquist number. Although a finite but small resistivity, required for numerical stability, is included in the linearized mode equations, the numerical and the analytical results are in good qualitative agreement.

\section{CONCLUSION}

With the help of a dissipationless MHD model that includes the effect of electron inertia, it has been shown that a sheared velocity field in a 2-D equilibrium configuration with a magnetic inversion line modifies the onset conditions and the growth rate of the collisionless kink mode. In particular, the sheared velocity field is able to stabilize partially the kink mode and to lower the value of the growth rate at which reconnection proceeds. This stabilization does not depend on whether the velocity field is parallel or antiparallel to the magnetic field, but only on the absolute value of the gradient of the equilibrium velocity compared to the gradient of the inverted magnetic field component inside the reconnecting layer.

When the absolute value of ratio between the equilibrium velocity gradient and the Alfvén velocity gradient (associated with the inverted magnetic field component), inside the reconnecting layer, is equal to 1 , the collisionless kink mode is totally stabilized. In particular, when this ratio approaches 1 , the use of the constant- $\psi$ approximation is valid also in the kink regime. This allows us to show the total kink stabilization explicitly (see Appendix).

When the ratio between the equilibrium velocity gradient and the Alfvén velocity gradient is greater than 1, the system usually becomes Kelvin-Helmholtz unstable. In that case, the reconnection occurring during the development of the Kelvin-Helmholtz instability is thus driven by the faster ideal instability. ${ }^{18-22}$

\section{APPENDIX: SLOW GROWTH REGIME}

Let us consider the slow growth regime, where $\gamma / \alpha F^{\prime}(0) \epsilon \ll 1$, in the "comparable shear" regime, where $\mid G^{\prime}(0) / F^{\prime}(0) \sim 1$. For the sake of simplicity, we take

$$
F^{\prime \prime}(0)=G^{\prime \prime}(0)=0, \quad F^{\prime}(0)>0, \quad G^{\prime}(0)>0 .
$$

From Eqs. (12) and (13) that describe the plasma dynamics inside the internal layer, we see that the only way to balance the different terms is to consider, once again, $d_{e}^{2} / \epsilon^{2} \sim 1$. From the use of the frozen-in law inside the internal region (11) and remembering that $\left|G^{\prime}(0) / F^{\prime}(0)\right|=O(1)$, we can estimate the relative importance of $\psi$ and $W$

$$
\psi \sim \frac{i \alpha F^{\prime}(0) \epsilon}{\gamma+i \alpha G^{\prime}(0) \epsilon} W \Rightarrow \psi \sim W .
$$

This estimate is different from the standard stationary plasma estimate. ${ }^{7}$ This is not surprising since, in our case, the convective term $G^{\prime}(0) / F^{\prime}(0)$ is far more important that the time derivative term $\gamma / \alpha F^{\prime}(0) \epsilon$.

In order to obtain the growth rate, we can consider the power expansions of the solution in the small parameter $\gamma / \alpha F^{\prime}(0) \epsilon$ 


$$
W=\sum_{n=0}^{\infty} W_{n}\left[\gamma /\left(\alpha F^{\prime}(0) \epsilon\right)\right]^{n}, \quad \psi=\sum_{n=0}^{\infty} \psi_{n}\left[\gamma /\left(\alpha F^{\prime}(0) \epsilon\right)\right]^{n} .
$$

The zeroth order of Eqs. (12) and (13) in the small parameter $\gamma / \alpha F^{\prime}(0) \epsilon$ gives us

$$
\begin{aligned}
& i \frac{G^{\prime}(0)}{F^{\prime}(0)} \xi \frac{\partial^{2} W_{0}}{\partial \xi^{2}}=i \xi \frac{\partial^{2} \psi_{0}}{\partial \xi^{2}}, \\
& i \frac{G^{\prime}(0)}{F^{\prime}(0)} \xi \psi_{0}-i \xi W_{0}=i \frac{G^{\prime}(0)}{F^{\prime}(0)} \xi \frac{\partial^{2} \psi_{0}}{\partial \xi^{2}},
\end{aligned}
$$

where we have taken $\epsilon=d_{e}$. The constant- $\psi$ solution

$$
\frac{G^{\prime}(0)}{F^{\prime}(0)} \psi_{0}=W_{0}=\frac{G^{\prime}(0)}{F^{\prime}(0)} C_{0}
$$

is the only one able to match the external solution. As in the case of resistive reconnection in the presence of an equilibrium velocity field, ${ }^{14,15,17}$ the collisionless "small growth" (comparable shear) regime corresponds to the constant- $\psi$ regime. At first order in the small parameter $\gamma / \alpha F^{\prime}(0) \epsilon$, we obtain

$$
\begin{aligned}
& i \frac{G^{\prime}(0)}{F^{\prime}(0)} \xi \frac{\partial^{2} W_{1}}{\partial \xi^{2}}=i \xi \frac{\partial^{2} \psi_{1}}{\partial \xi^{2}}, \\
& \psi_{0}+i \frac{G^{\prime}(0)}{F^{\prime}(0)} \xi \psi_{1}-i \xi W_{1}=i \frac{G^{\prime}(0)}{F^{\prime}(0)} \xi \frac{\partial^{2} \psi_{1}}{\partial \xi^{2}} .
\end{aligned}
$$

It is noteworthy that Eq. (A8) is singular in $\xi=0$. This singularity is related, once again, to the existence of the continuous spectrum. ${ }^{32-34}$ As stated before, its contribution to the perturbation evolution is proportional to $t^{-|a|}$ and is thus negligible compared to the exponential instability. Furthermore, we will see that the logarithmic singularity in zero is of the form $q \ln q$, and thus will not pose any problem in founding the discrete spectrum. The matching with the external solution imposes $\psi_{1} \rightarrow 0$ for $|\xi| \gg 1$, and thus, from Eqs. (A7) and (A8), we obtain

$$
\begin{aligned}
& W_{1} \frac{G^{\prime}(0)}{F^{\prime}(0)}=\psi_{1}, \\
& \frac{G^{\prime}(0)}{F^{\prime}(0)} \psi_{0}-i \xi\left\{1-\left[\frac{G^{\prime}(0)}{F^{\prime}(0)}\right]^{2}\right\} \psi_{1}=i\left[\frac{G^{\prime}(0)}{F^{\prime}(0)}\right]^{2} \xi \frac{\partial^{2} \psi_{1}}{\partial \xi^{2}} .
\end{aligned}
$$

The last equation can be rewritten as

$$
\xi \frac{\partial^{2} \psi_{1}}{\partial \xi^{2}}+K^{2} \xi \psi_{1}+I=0
$$

with $K^{2}=\left\{1-\left[G^{\prime}(0) / F^{\prime}(0)\right]^{2}\right\} /\left[G^{\prime}(0) / F^{\prime}(0)\right]^{2}$ and $I=i \psi_{0}$ $\times\left[G^{\prime}(0) / F^{\prime}(0)\right]$.
If we assume that $\left\{1-\left[G^{\prime}(0) / F^{\prime}(0)^{2}\right)<0\right.$ and

$$
K=-i\left\{\frac{\left\{\left[G^{\prime}(0) / F^{\prime}(0)\right]^{2}-1\right\}}{\left[G^{\prime}(0) / F^{\prime}(0)\right]^{2}}\right\}^{1 / 2},
$$

the solution of Eq. (A11), compatible with the boundary conditions, is given by

$$
\psi_{1}(\xi)=-\frac{I}{K}[\operatorname{ci}(K \xi) \sin (K \xi)-s i(K \xi) \cos (K \xi)],
$$

where

$$
s i(K \xi)=-\int_{K \xi}^{+\infty} \frac{\sin q}{q} d q, \quad c i(K \xi)=-\int_{K \xi}^{+\infty} \frac{\cos q}{q} d q .
$$

The functions $s i, c i$ can be written using the functions integral sine $\mathrm{Si}$ and integral cosine $\mathrm{Cin}$, analytic over the whole complex plane

$$
\begin{aligned}
& \operatorname{si}(q)=\operatorname{Si}(q)-\pi / 2, \\
& \operatorname{ci}(q)=C+\ln q-\operatorname{Cin}(q), \quad \arg q \in[-\pi, \pi], \\
& \operatorname{Cin}(-q)=\operatorname{Cin}(q), \\
& \operatorname{Si}(-q)=-\operatorname{Si}(q) .
\end{aligned}
$$

The asymptotic behavior of $\mathrm{Cin}$ and $\mathrm{Si}$ is given by

$$
S i(x) \simeq \frac{\pi}{2}-\frac{\cos x}{x}\left(1-\frac{2 !}{x^{2}}+\ldots\right)-\frac{\sin x}{x}\left(\frac{1}{x}-\frac{3 !}{x^{3}}+\ldots\right),
$$

$$
\begin{aligned}
\operatorname{Cin}(x) \simeq & C+\ln x-\frac{\sin x}{x}\left(1-\frac{2 !}{x^{2}}+\ldots\right) \\
& +\frac{\cos x}{x}\left(\frac{1}{x}-\frac{3 !}{x^{3}}+\ldots\right),
\end{aligned}
$$

for $\operatorname{Re} x>0$. Thus we have, for $|\xi| \gg 1, \operatorname{Im} \xi>0$,

$$
\psi_{1}(\xi) \simeq-i \frac{\psi_{0}}{1-\left[G^{\prime}(0) / F^{\prime}(0)\right]^{2}}\left[\frac{G^{\prime}(0)}{F^{\prime}(0)}\right] \frac{1}{\xi} .
$$

It is thus necessary to consider the second order terms in the small parameter $\gamma / \alpha F^{\prime}(0) \epsilon$ in order to match the internal and external solutions. At this order Eqs. (12) and (13) become

$$
\begin{aligned}
& \frac{\partial^{2} W_{1}}{\partial \xi^{2}}+i \frac{G^{\prime}(0)}{F^{\prime}(0)} \xi \frac{\partial^{2} W_{2}}{\partial \xi^{2}}=i \xi \frac{\partial^{2} \psi_{2}}{\partial \xi^{2}}, \\
& \psi_{1}+i \frac{G^{\prime}(0)}{F^{\prime}(0)} \xi \psi_{2}-i \xi W_{2}=i \frac{G^{\prime}(0)}{F^{\prime}(0)} \xi \frac{\partial^{2} \psi_{2}}{\partial \xi^{2}} .
\end{aligned}
$$

From Eq. (A23) we obtain

$$
\int_{-\infty}^{+\infty} \frac{\partial^{2} W_{2}}{\partial \xi^{2}} d \xi=\frac{G^{\prime}(0)}{F^{\prime}(0)} \int_{-\infty}^{+\infty} \frac{\partial^{2} \psi_{2}}{\partial \xi^{2}} d \xi
$$


If we insert Eq. (A24) into Eq. (A22) and integrate, it yields

$$
\left[\frac{G^{\prime}(0)}{F^{\prime}(0)}\right]^{2} \int_{-\infty}^{+\infty} \frac{\partial^{2} \psi_{2}}{\partial \xi^{2}} d \xi=\int_{-\infty}^{+\infty} \frac{\partial^{2} \psi_{2}}{\partial \xi^{2}} d \xi+i \int_{-\infty}^{+\infty} \frac{1}{\xi} \frac{\partial^{2} W_{1}}{\partial \xi^{2}} d \xi
$$

From the definition of $\Delta^{\prime}$

$$
\int_{-\infty}^{+\infty} \frac{\partial^{2} \psi_{2}}{\partial \xi^{2}} d \xi=\frac{\epsilon \Delta^{\prime} \psi_{0}}{\left[\gamma /\left(\alpha F^{\prime}(0) \epsilon\right)\right]^{2}},
$$

we have

$$
\begin{gathered}
\left\{\left[\frac{G^{\prime}(0)}{F^{\prime}(0)}\right]^{2}-1\right\} \frac{\epsilon \Delta^{\prime} \psi_{0}}{\left\{\gamma\left[\alpha F^{\prime}(0) \epsilon\right]\right\}^{2}} \\
=\int_{-\infty}^{+\infty} \frac{\frac{G^{\prime}(0)}{F^{\prime}(0)} \psi_{0}+i \xi\left[\left(\frac{G^{\prime}(0)}{F^{\prime}(0)}\right)^{2}-1\right] \psi_{1}}{\left[G^{\prime}(0) / F^{\prime}(0)\right]^{3} \xi^{2}} .
\end{gathered}
$$

Thanks to the asymptotic behavior of $\psi_{1}$ and to its behavior at $\xi=0$, we can close the integral path in the upper half of the complex plane and consider the indentation around $\xi=0$. As stated before, the logarithmic singularity at $\xi=0$ does not give any contribution to our mode. In fact we obtain

$$
\begin{aligned}
\frac{\epsilon \Delta^{\prime} \psi_{0}}{\left(\frac{\gamma}{\alpha F^{\prime}(0) \epsilon}\right)^{2}} & =i \pi \frac{\left.i \psi_{1}\right|_{\xi=0}}{\left(\frac{G^{\prime}(0)}{F^{\prime}(0)}\right)^{3}} \\
& =-\frac{\pi^{2}}{2} \psi_{0}\left|\frac{G^{\prime}(0)}{F^{\prime}(0)}\right|^{-3}\left\{\left[\frac{G^{\prime}(0)}{F^{\prime}(0)}\right]^{2}-1\right\}^{-1 / 2},
\end{aligned}
$$

which yields the following expression for the growth rate:

$$
\gamma^{2}=-\frac{2}{\pi^{2}} \alpha^{2}\left[F^{\prime}(0)\right]^{2} d_{e}^{3} \Delta^{\prime}\left|\frac{G^{\prime}(0)}{F^{\prime}(0)}\right|^{3}\left\{\left[\frac{G^{\prime}(0)}{F^{\prime}(0)}\right]^{2}-1\right\}^{1 / 2} .
$$

We note that the scaling of the growth rate is different from that of the tearing mode in a stationary plasma. Furthermore, the small growth mode is stable for $\left[G^{\prime}(0) / F^{\prime}(0)\right]^{2}>1$ and $\Delta^{\prime}>0$ (while $\Delta^{\prime}>0$ is the usual tearing instability condition). It is important to note that usually the system is Kelvin-Helmholtz unstable for $\left[G^{\prime}(0) / F^{\prime}(0)\right]^{2}>1$, thus the tearing instability for $\Delta^{\prime}<0$ has a growth rate negligible with respect to that of the faster ideal Kelvin-Helmholtz instability. $^{14-18}$ From the assumption $\gamma / \alpha F^{\prime}(0) \epsilon \ll 1$ and from Eq. (A29), we obtain $\Delta^{\prime} \epsilon\left\{\left[G^{\prime}(0) / F^{\prime}(0)\right]^{2}-1\right\}^{1 / 2} \ll 1$, which justifies the constant- $\psi$ approximation for $\Delta^{\prime} \sim 1$.

However, it is important to observe that the constant- $\psi$ approximation is also justified for $\Delta^{\prime} \gg 1$ when $\left[G^{\prime}(0) / F^{\prime}(0)\right]^{2} \rightarrow 1$. We can thus state that the $\Delta^{\prime} \gg 1$ Kink mode is totally stabilized by the equilibrium velocity field when $\left[G^{\prime}(0) / F^{\prime}(0)\right]^{2} \rightarrow 1$.

${ }^{1}$ S. Chandrasekhar, Hydrodynamic and Hydromagnetic Stability (Oxford University Press, New York, 1961), p. 481.

${ }^{2}$ A. Miura, Phys. Rev. Lett. 49, 779 (1982).

${ }^{3}$ C. D. Winant and F. K. Browand, J. Fluid Mech. 63, 237 (1974).

${ }^{4}$ F. K. Browand and P. D. Weidman, J. Fluid Mech. 76, 127 (1976).

${ }^{5}$ A. Miura, Phys. Plasmas 4, 2871 (1997).

${ }^{6}$ J. Dungey, Phys. Rev. Lett. 6, 47 (1961).

${ }^{7}$ H. P. Furth, J. Killeen, and M. N. Rosenbluth, Phys. Fluids 6, 459 (1963).

${ }^{8}$ P. A. Sturrock and B. Coppi, Nature (London) 204, 61 (1964).

${ }^{9}$ M. E. Mandt, R. E. Denton, and J. F. Drake, Geophys. Res. Lett. 21, 73, doi:10.1029/93GL03382 (1994).

${ }^{10}$ M. A. Shay, J. F. Drake, R. E. Denton, and D. Biskamp, J. Geophys. Res. 103, 9165, doi:10.1029/97JA03528 (1998).

${ }^{11}$ G. Vekstein and N. H. Bian, Phys. Plasmas 13, 122105 (2006).

${ }^{12}$ M. Faganello, F. Califano, and F. Pegoraro, Phys. Rev. Lett. 101, 175003 (2008).

${ }^{13}$ M. Faganello, F. Califano, and F. Pegoraro, New J. Phys. 11, 063008 (2009).

${ }^{14}$ I. Hofman, Plasma Phys. 17, 143 (1975).

${ }^{15}$ M. Dobrowolny, J. Plasma Phys. 29, 393 (1983).

${ }^{16}$ G. Einaudi and F. Rubini, Phys. Fluids 29, 2563 (1986).

${ }^{17}$ X. L. Chen and P. J. Morrison, Phys. Fluids B 2, 495 (1990).

${ }^{18}$ Q. Chen, A. Otto, and L. C. Lee, J. Geophys. Res. 102, 151, doi:10.1029/ 96JA03144 (1997).

${ }^{19}$ Z. X. Liu and Y. D. Hu, Geophys. Res. Lett. 15, 752, doi:10.1029/ GL015i008p00752 (1988).

${ }^{20}$ D. A. Knoll and L. Chacón, Phys. Rev. Lett. 88, 215003 (2002).

${ }^{21}$ T. K. M. Nakamura and M. Fujimoto, Adv. Space Res. 37, 522 (2006).

${ }^{22}$ F. Califano, M. Faganello, F. Pegoraro, and F. Valentini, Nonlinear Processes Geophys. 16, 1 (2009).

${ }^{23}$ A. Otto and D. H. Fairfield, J. Geophys. Res. 105, 21175, doi:10.1029/ 1999JA000312 (2000).

${ }^{24}$ H. Baty, R. Keppens, and P. Compte, Phys. Plasmas 10, 4661 (2003).

${ }^{25}$ T. K. M. Nakamura and M. Fujimoto, Geophys. Res. Lett. 32, L21102, doi:10.1029/2005GL023362 (2005).

${ }^{26}$ M. Faganello, F. Califano, and F. Pegoraro, Phys. Rev. Lett. 101, 105001 (2008).

${ }^{27}$ B. Coppi, Phys. Lett. 11, 226 (1964).

${ }^{28}$ J. A. Wesson, Nucl. Fusion 30, 2545 (1990).

${ }^{29}$ P. J. Morrison, Phys. Lett. A 80, 383 (1980).

${ }^{30}$ M. Ottaviani and F. Porcelli, Phys. Rev. Lett. 71, 3802 (1993).

${ }^{31}$ D. Grasso, F. Pegoraro, F. Porcelli, and F. Califano, Plasma Phys. Controlled Fusion 41, 1497 (1999).

${ }^{32}$ K. M. Case, Phys. Fluids 3, 143 (1960).

${ }^{33}$ E. M. Barston, Ann. Phys. (N.Y.) 29, 282 (1964).

${ }^{34}$ A. Hasegawa and C. Uberoi, The Alfvèn Wave (U.S. Department of Energy, Oak Ridge, 1982), p. 33. 